\title{
Meglitinide Antidiabetic Agent
}

National Cancer Institute

\section{Source}

National Cancer Institute. Meglitinide Antidiabetic Agent. NCI Thesaurus. Code C98079.

Nonsulfonylurea insulin secretagogues and analogues of meg litinide with antihyperglycemic activity. Meglitinide antidiabetic agents bind to the ATP-sensitive inwardly-rectifier potassium (IKATP) channels on the membranes of pancreatic beta cells in a manner similar to sulfonylureas but at a discrete contact point on the SUR1 subunit. The binding blocks the influx of positively charged potassium ions into the cells, thereby reducing potassium conductance and causing membrane depolarization. In turn, this stimulates calcium ion influx through voltage-gated calcium channels, raising intracellular calcium ion levels and leading to the release of insulin-containing granules The IKATP channels found in pancreatic islets are complexes of four IKATP 6.2 and four SUR1 subunits. Compared to sulfonylureas, meglitinides, in general, have a more rapid onset of action and a shorter half-life. 